\title{
Error Probabilities and Threshold Selection in Networked Nuclear Detection
}

\author{
Chetan D. Pahlajani, Jianxin Sun, Ioannis Poulakakis, Herbert G. Tanner
}

\begin{abstract}
We consider the problem of computing analytical bounds on error probabilities in the setting of networked nuclear detection based on a likelihood ratio test. The detection scenario involves a mobile source of known trajectory passing within the sensing range of a spatially distributed sensor array, which has to decide on its nature (benign or radioactive) within a fixed time interval. Exploiting the particular modeling structure of remote nuclear measurement, and the form that the likelihood ratio takes in this setting, the paper presents analytical Chernoff bounds for the error probabilities, which in turn allow the selection of threshold constants for the likelihood ratio test in a computationally efficient manner compared to Monte Carlo simulations.
\end{abstract}

Keywords - Nuclear detection; Likelihood Ratio Test; Chernoff bounds.

\section{INTRODUCTION}

An important aspect of nuclear detection relates to the problem of using radiation sensor data to decide, within a given time interval, whether a radioactive source is present in the vicinity of the sensor(s). This problem is of significance not only in the context of national security, but also nuclear nonproliferation in general. Specifically, it is noted that currently deployed systems for detecting the transport of illicit radioactive material are not adequate [1]. The use of networks of sensors is among the proposed suite of approaches that could constitute an integrated solution.

The problem set-up in this paper involves a suspect target, which may or may not be radioactive, which passes through a finite, spatially distributed array of radiation sensors. Being mobile, the target is in the vicinity of the sensor array for a limited amount of time, in which the sensors have to decide on its nature. The motion of the target is assumed known or at least measurable accurately enough for its position to be described as a deterministic function of time. The target is considered as a source if its radioactivity is estimated above a given level, and the decision problem that arises is how to utilize the information gathered at individual sensors so that a global decision can be made in a timely manner.

Assuming that the sources of radiation are concealed, their presence is ascertained based on their nuclear emissions. Such emissions are modeled as discrete events corresponding to a Poisson point process, i.e. the time interval between consecutive emissions of particles or gamma rays is expo-

Chetan Pahlajani is with the Department of Mathematical Sciences at the University of Delaware, Newark DE 19716; E-mail: chetan@math. udel.edu.

Jianxin Sun, Ioannis Poulakakis and Bert Tanner are with the Department of Mechanical Engineering, at the University of Delaware, Newark DE 19716; E-mail: $\{j x s u n$, poulakas, btanner\}@udel.edu. nentially distributed [2]. ${ }^{1}$ In the case considered here, the relative motion between the source and sensors causes the point processes describing the arrival of gamma rays at the sensors to be time inhomogeneous. Another challenge is that the signal recorded by the radiation detectors contains, in addition to possible emissions from the suspected source, a background radiation component due to cosmic radiation and naturally occurring radioactive material. It is also highly likely that an illicit radioactive source, if present, is shielded, making its perceived intensity at the sensors comparable in magnitude to that of background radiation. In addition, this perceived intensity drops with the square of the distance between sensor and source [2]. To summarize, sensors need to detect from a distance, a Poisson signal which is buried inside another Poisson signal of similar nature and magnitude, within a short time interval.

Aspects of the problem of deciding between two hypotheses regarding point processes, one corresponding to a message "all clear" and the other to "alarm," are studied in [3], [7]-[11]. The common idea in all these works is finding the right likelihood ratio, computing it based on the sensor data, and comparing this ratio with a carefully selected threshold. Depending on whether the ratio is above or below the threshold, one of the two hypotheses is accepted; this comparison is known as the likelihood ratio test (LR test). In the specific context of radiation detection networks, related work is based on adaptations of the LR test, using either sequential or fixed-interval testing theory [12], [13], and Bayesian or Neyman-Pearson approaches [2], [14], [15]. With the exception of [13], sensor observations have been assumed independent identically distributed (i.i.d.).

In an LR test, there are two errors that can be made: a false alarm, where the test decides that a source is present when in fact the "all clear" hypothesis is correct, and a missed detection in which the test shows "all clear" but a source is indeed present. Computing the error probabilities analytically is impractical, and thus upper and lower bounds are sought, and efforts to this end have been made using Chernoff bounds [10], [16]-[18]. The use of Chernoff bounds (and modifications) in performance evaluation of communications systems is considered in [19]-[22], while connections to the theory of large deviations are explored in [23]-[25].

This paper leverages recent results [26] regarding the fixed-time interval detection of weak time-inhomogeneous Poisson signals buried in (also) Poisson carrier background, using a network of detectors capable of performing some

\footnotetext{
${ }^{1}$ Poisson processes are common in other domains too, such as queueing theory [3], [4], optical communications systems [5], and the generation of electrical pulses in neurons [6].
} 
limited local computation. The main conclusion of [26] (see Section III), is that the likelihood ratio used in a threshold test for detection can be computed as a direct product of factors computed locally at sensors. As a result, with decentralized local computation, the performance of centralized detection can be matched without either local decisions at the sensor level, or streaming the entirety of raw sensor data to some designated fusion center. The present paper takes a step forward in this direction, deriving analytical Chernoff bounds on the probabilities of missed detection and false alarm, which allow the selection of threshold constants for the LR test in a more efficient manner compared to Monte Carlotype analysis.

\section{PROBLEM DESCRIPTION}

Consider a planar (for simplicity) array of $k$ radiation sensors. The location of sensor $i, 1 \leq i \leq k$, is expressed by the pair $\left(x_{i}, y_{i}\right)$. A suspected source of intensity $a>0$ moves in the $(x, y)$ plane with position given by $\left(x_{s}(t), y_{s}(t)\right)$.

Let $r_{i}(t)$ be the distance between the source and sensor $i$ at time $t$. Thus,

$$
r_{i}(t)=\sqrt{\left(x_{s}(t)-x_{i}\right)^{2}+\left(y_{s}(t)-y_{i}\right)^{2}} .
$$

The intensity due to the source as seen at sensor $i$ is modeled by $\nu_{i}(t)=\frac{\chi a}{\chi+r_{i}(t)^{2}}$, where $\chi$ is a sensor-specific crosssection coefficient. If $\beta_{i}(t)$ is the background activity at the location of sensor $i$ (assumed known), the question is to decide whether the sensor observations are governed by the intensities $\beta_{i}(t)$ or $\beta_{i}(t)+\nu_{i}(t), 1 \leq i \leq k$, corresponding to the target being benign versus radioactive, respectively.

\section{PRELIMINARIES}

In this section, we recall the setup from [26], which provides the starting point for the analysis in this work. Consider the following detection problem: a network of $k$ spatially distributed sensors observes a time-inhomogeneous Poisson process and has to decide - at the end of a fixed time interval $[0, T]$-between two hypotheses, $H_{0}$ and $H_{1}$, regarding the statistics of the observed process. To this end, each sensor transmits at time $T$ a locally processed summary of its information in the form of a likelihood ratio-not a decision-to a fusion center, which then combines these messages to arrive at an optimal decision in the NeymanPearson framework. The resulting test compares the (global) likelihood ratio $L_{T}$ against a threshold $\gamma>0$, deciding $H_{1}$ if $L_{T} \geq \gamma$, and $H_{0}$ otherwise.

The probabilistic setup is as follows. We have a measurable space $(\Omega, \mathscr{F})$, on which a $k$-dimensional vector of counting processes $N_{t}=\left(N_{t}(1), \ldots, N_{t}(k)\right), t \in[0, T]$ is defined. For the problem at hand, $N_{t}(i)$ represents the number of counts registered at sensor $i \in\{1,2, \ldots, k\}$ up to (and including) time $t \in[0, T]$. The two hypotheses $H_{0}$ and $H_{1}$ regarding the state of the environment correspond to two distinct probability measures on $(\Omega, \mathscr{F})$. Hypothesis $H_{0}$ corresponds to a probability measure $\mathbb{P}_{0}$, with respect to which the $N_{t}(i), 1 \leq i \leq k$, are independent Poisson processes over $t \in[0, T]$ with intensities $\beta_{i}(t)$, respectively.
Hypothesis $H_{1}$ corresponds to a probability measure $\mathbb{P}_{1}$, with respect to which the $N_{t}(i), 1 \leq i \leq k$, are independent Poisson processes over $t \in[0, T]$ with intensities $\beta_{i}(t)+$ $\nu_{i}(t)$, respectively. The decision problem is thus one of identifying the correct probability measure $\left(\mathbb{P}_{0}\right.$ versus $\left.\mathbb{P}_{1}\right)$ on $(\Omega, \mathscr{F})$, based on a realization of the $k$-dimensional process $N_{t}=\left(N_{t}(1), \ldots, N_{t}(k)\right)$.

Analysis motives the following requirements on $\beta_{i}$ and $\nu_{i}$ :

Assumption 1: For $1 \leq i \leq k, \beta_{i}:[0, T] \rightarrow\left[\beta_{\min }, \beta_{\max }\right]$ is a bounded, continuous function with $0<\beta_{\min }<\beta_{\max }<$ $\infty, \beta_{\min }, \beta_{\max }$ independent of $i \in\{1,2, \ldots, k\}$.

Assumption 2: For $1 \leq i \leq k, \nu_{i}:[0, T] \rightarrow\left[\nu_{\min }, \nu_{\max }\right]$ is a bounded, continuous function with $0<\nu_{\min }<\nu_{\max }<\infty$, $\nu_{\min }, \nu_{\max }$ independent of $i \in\{1,2, \ldots, k\}$.

Let $\left(\mathscr{F}_{t}^{N}: 0 \leq t \leq T\right)$ be the filtration generated by the process $N_{t} \cdot{ }^{2}$ Then, for any event $A \in \mathscr{F}_{t}^{N}$, an observer of the sample path $s \mapsto N_{s}, 0 \leq s \leq t$, knows at time $t$ whether or not the event $A$ has occurred. The $\sigma$-field $\mathscr{F}_{T}^{N}$ thus represents the information generated by the totality of sensor observations up to $t=T$; to wit, the information on which the decision must be based.

A test for deciding between hypotheses $H_{0}$ and $H_{1}$ on the basis of $\mathscr{F}_{T}^{N}$ observations can be thought of as a set $A_{1} \in \mathscr{F}_{T}^{N}$ with the following significance: if the outcome $\omega \in A_{1}$, decide $H_{1}$; if $\omega \in A_{0} \triangleq \Omega \backslash A_{1}$, decide $H_{0}$. For a test $A_{1} \in \mathscr{F}_{T}^{N}$, two types of errors might occur. A "false alarm" occurs when the outcome $\omega \in A_{1}$ (i.e. decide $H_{1}$ ) while $H_{0}$ is the correct hypothesis. A "miss" occurs when $\omega \in \Omega \backslash A_{1}$ (i.e. decide $H_{0}$ ) while $H_{1}$ is the correct hypothesis. Clearly, the probability of false alarm is given by $\mathbb{P}_{0}\left(A_{1}\right)$, while the probability of a miss is given by $\mathbb{P}_{1}(\Omega \backslash$ $\left.A_{1}\right)$. Then, the probability of detection is given by $\mathbb{P}_{1}\left(A_{1}\right)=$ $1-\mathbb{P}_{1}\left(\Omega \backslash A_{1}\right)$.

In the Neyman-Pearson framework, one is given an acceptable upper bound on the probability of false alarm $\alpha \in(0,1)$, and the problem is to find an optimal test: a set $A_{1}^{*} \in \mathscr{F}_{T}^{N}$ which maximizes the probability of detection over all tests whose probability of false alarm is less than or equal to $\alpha$. The following result provides an optimal test that employs local information processing at the sensor level, to enable decisions at the fusion center that recover the optimal performance of a centralized Neyman-Pearson test. The underlying probabilistic setup is as described above.

Theorem 1 ([26]): Consider a network with $k$ sensors and a fusion center connected in a parallel configuration. For $1 \leq i \leq k$, let $N_{t}(i), t \in[0, T]$ denote the observation at sensor $i$ over the time interval $[0, T]$ and let $\left(\tau_{n}(i): n \geq 1\right)$ be the jump times of $N_{t}(i)$. Assume that at decision time $T$, sensor $i$ transmits to the fusion center the statistic

$$
L_{T}(i) \triangleq \exp \left(-\int_{0}^{T} \nu_{i}(s) d s\right) \prod_{n=1}^{N_{T}(i)}\left(1+\frac{\nu_{i}\left(\tau_{n}(i)\right)}{\beta_{i}\left(\tau_{n}(i)\right)}\right)
$$

computed on the basis of its observation $t \mapsto N_{t}(i), t \in$

\footnotetext{
${ }^{2}$ Definition: for $t \in[0, T], \mathscr{F}_{t}^{N}=\sigma\left(N_{s}: 0 \leq s \leq t\right)$ is the smallest $\sigma$-field on $\Omega$ with respect to which all the ( $k$-dimensional) random variables $N_{s}, 0 \leq s \leq t$, are measurable.
} 
$[0, T]$. Then, the test $A_{1}^{*}=\left\{L_{T} \geq \gamma\right\}$ performed at the fusion center, with $L_{T} \triangleq \prod_{i=1}^{k} L_{T}(i)$, and $\gamma>0$ satisfying $\mathbb{P}_{0}\left(L_{T} \geq \gamma\right)=\alpha$, is optimal for $\mathscr{F}_{T}^{N}$-observations in the sense that for any $A_{1} \in \mathscr{F}_{T}^{N}$ with $\mathbb{P}_{0}\left(A_{1}\right) \leq \alpha$, we have $\mathbb{P}_{1}\left(A_{1}^{*}\right) \geq \mathbb{P}_{1}\left(A_{1}\right)$.

Remark 1: Note that the threshold $\gamma$ in Theorem 1 may not exist for every $\alpha \in(0,1) .^{3}$ The classical NeymanPearson result covers such cases by allowing tests with randomization [27], [28]. However, what is actually required for the problem of nuclear detection is that $\alpha$ be of a certain order of magnitude, rather than a specific number (for instance, one might require that $\alpha$ be of order $10^{-4}$ ). Therefore, we will not consider randomized test, and assume in the sequel that we have the flexibility to first choose $\gamma$, with the proviso that $\alpha=\mathbb{P}_{0}\left(L_{T} \geq \gamma\right)$ is of the right order of magnitude.

\section{Chernoff Bounds on ERror Probabilities}

The performance of the test $\left\{L_{T} \geq \gamma\right\}$ can be measured by the probabilities of false alarm and miss defined by

$$
P_{F} \triangleq \mathbb{P}_{0}\left(L_{T} \geq \gamma\right) \quad \text { and } \quad P_{M} \triangleq \mathbb{P}_{1}\left(L_{T}<\gamma\right)
$$

respectively. In many cases of interest, however, the exact computation of these error probabilities is mathematically intractable, motivating the need for good upper bounds which are easily computable. Theorem 2, stated below, derives explicitly computable bounds on $P_{F}$ and $P_{M}$ based on Chernoff's inequalities. To state the theorem, we define

$$
\Lambda(p) \triangleq \log \mathbb{E}_{0}\left[L_{T}^{p}\right]=\log \mathbb{E}_{0}\left[\mathrm{e}^{p \ell_{T}}\right],
$$

where $\ell_{T} \triangleq \log L_{T}$ is the $\log$ likelihood ratio. Thus $\Lambda(p)$ is the cumulant generating function of $\ell_{T}$ under $H_{0}$. Let

$$
\mu_{i}(t) \triangleq 1+\frac{\nu_{i}(t)}{\beta_{i}(t)}
$$

for $1 \leq i \leq k, t \in[0, T]$; thus, $\mu_{i}(t)$ is the ratio of intensities for $H_{1}$ versus $H_{0}$. The intensities at sensor $i$ at time $t$ under $H_{0}$ and $H_{1}$ can now be written as $\beta_{i}(t), \mu_{i}(t) \beta_{i}(t)$, respectively. The proof of the following result is at the end of the section.

Theorem 2: Let $\eta \triangleq \log \gamma \in \mathbb{R}$. The Chernoff bounds on $P_{F}$ and $P_{M}$ are given by

$$
\begin{aligned}
& P_{F} \leq \exp \left(\inf _{p>0}[\Lambda(p)-p \eta]\right) \\
& P_{M} \leq \exp \left(\inf _{p<1}[\Lambda(p)+(1-p) \eta]\right)
\end{aligned}
$$

where $\Lambda(p)$ is explicitly computable via

$$
\Lambda(p)=\sum_{i=1}^{k} \int_{0}^{T}\left[\mu_{i}(s)^{p}-p \mu_{i}(s)+p-1\right] \beta_{i}(s) d s
$$

for $p \in \mathbb{R}$.

\footnotetext{
${ }^{3}$ If one were dealing instead with a continuous random variable $Z$ admitting a density $f(x)$, one could always find $\gamma$ by solving $\int_{\gamma}^{\infty} f(x) d x=$ $\alpha$.
}

Remark 2: The explicit analytical formula for $\Lambda(p)$ in (6) is a consequence of the assumption of deterministic intensities, which allows us to circumvent the intricate filtering analysis in [10] needed to accommodate stochastic intensities.

We prove Theorem 2 through a series of steps. The principal result in this direction is Proposition 1, stated next, whose application at $t=T$, in conjunction with (3), yields (6). To start, we introduce a stochastic process whose value at time $T$ is the likelihood ratio $L_{T}$. Let $\left(L_{t}: t \in[0, T]\right)$ be the process

$$
L_{t} \triangleq \prod_{i=1}^{k}\left\{\exp \left(\int_{0}^{t}\left[1-\mu_{i}(s)\right] \beta_{i}(s) d s\right) \prod_{n=1}^{N_{t}(i)} \mu_{i}\left(\tau_{n}(i)\right)\right\} \text {, }
$$

where by convention, $\prod_{n=1}^{0}(\cdot)=1$.

Proposition 1: For any $p \in \mathbb{R}, t \in[0, T]$, we have

$$
\mathbb{E}_{0}\left[L_{t}^{p}\right]=\exp \left(\sum_{i=1}^{k} \int_{0}^{t}\left[\mu_{i}(s)^{p}-p \mu_{i}(s)+p-1\right] \beta_{i}(s) d s\right) .
$$

Proposition 1 is proved using Lemmas 1 and 2 below, whose proofs are given in the Appendix.

Lemma 1: For any $p \in \mathbb{R}, t \in[0, T]$, it is $\mathbb{E}_{0}\left[L_{t}^{p}\right]<\infty$.

Lemma 2: For any $p \in \mathbb{R}, t \in[0, T]$, we have

$$
\begin{aligned}
L_{t}^{p} & =1+\sum_{i=1}^{k} \int_{0}^{t} L_{s-}^{p}\left[\mu_{i}(s)^{p}-1\right] d M_{s}(i) \\
& +\sum_{i=1}^{k} \int_{0}^{t} L_{s-}^{p}\left[\mu_{i}(s)^{p}-p \mu_{i}(s)+p-1\right] \beta_{i}(s) d s
\end{aligned}
$$

where $M_{t}(i) \triangleq N_{t}(i)-\int_{0}^{t} \beta_{i}(s) d s$.

Proof: [Proof of Proposition 1] Note that for each $1 \leq$ $i \leq k, \int_{0}^{t} L_{s-}^{p}\left[\mu_{i}(s)^{p}-1\right] d M_{s}(i)$ in (9) is a zero-mean $\mathbb{P}_{0^{-}}$ martingale by [3, Theorem II.3.T8]. ${ }^{4}$ Taking expectations in (9), we get

$$
\begin{gathered}
\mathbb{E}_{0}\left[L_{t}^{p}\right]= \\
1+\int_{0}^{t} \mathbb{E}_{0}\left[L_{s-}^{p}\right]\left(\sum_{i=1}^{k}\left[\mu_{i}(s)^{p}-p \mu_{i}(s)+p-1\right] \beta_{i}(s)\right) d s,
\end{gathered}
$$

where we have used Lemma 1 to interchange integral and expectation. The stated result now follows from [3, Theorem A4.T4] with $x(t)=\mathbb{E}_{0}\left[L_{t}^{p}\right], a(t)=t, u(t)=$ $\sum_{i=1}^{k}\left[\mu_{i}(t)^{p}-p \mu_{i}(t)+p-1\right] \beta_{i}(t)$.

Proof: [Proof of Theorem 2] Writing $\mathbb{E}_{0}\left[L_{T}^{p}\right]=\mathrm{e}^{\Lambda(p)}$, $\gamma=\mathrm{e}^{\eta}$, the Markov inequality together with Lemma 1 easily yields (5); see, for example [28, Section III.C.2]. Equation (6) is a direct consequence of (3) and Proposition 1 above.

\footnotetext{
${ }^{4}$ Equations (16) and (17), together with [3, Theorem I.3.T5], ensure that [3, Theorem II.3.T8] does in fact apply.
} 


\section{REALIZING THE PROBABILITY BOUNDS}

To effectively use Theorem 2, one needs to know where, if at all, the infima in (5) are realized. Moreover, for the bounds in (5) to be non-trivial, it is necessary that these infima be negative. Proposition 2 below assures us that if $\eta$ is chosen to lie in the open interval $\left(\Lambda^{\prime}(0), \Lambda^{\prime}(1)\right)$ (where $\left.\Lambda^{\prime}(0)<0<\Lambda^{\prime}(1)\right)$, then there is a unique $p^{*} \in(0,1)$ satisfying $\Lambda^{\prime}\left(p^{*}\right)=\eta$, for which both the infima in (5) are achieved and are negative.

To state Proposition 2, we introduce the following notation. Define the functions $\mathscr{E}_{F}, \mathscr{E}_{M}$ mapping $(0,1)$ to $\mathbb{R}$ by

$$
\begin{aligned}
& \mathscr{E}_{F}(p) \triangleq \Lambda(p)-p \Lambda^{\prime}(p) \\
& \mathscr{E}_{M}(p) \triangleq \Lambda(p)+(1-p) \Lambda^{\prime}(p) .
\end{aligned}
$$

Proposition 2: Fix $\eta=\log \gamma \in\left(\Lambda^{\prime}(0), \Lambda^{\prime}(1)\right)$. Then, there is a unique $p^{*} \in(0,1)$ given by $\Lambda^{\prime}\left(p^{*}\right)=\eta$ such that

$$
\begin{aligned}
\inf _{p>0}[\Lambda(p)-p \eta] & =\mathscr{E}_{F}\left(p^{*}\right)<0, \\
\inf _{p<1}[\Lambda(p)+(1-p) \eta] & =\mathscr{E}_{M}\left(p^{*}\right)<0 .
\end{aligned}
$$

The tightest error probability bounds for the decision test $\left\{L_{T} \geq \gamma\right\}$ are given by

$$
P_{F} \leq \exp \left[\mathscr{E}_{F}\left(p^{*}\right)\right], \quad P_{M} \leq \exp \left[\mathscr{E}_{M}\left(p^{*}\right)\right] .
$$

Proof: (sketch) Due to lack of space, we give an overview of the proof. One can use (6) to show that $\Lambda^{\prime}(0)<$ $0<\Lambda^{\prime}(1)$, and that $\Lambda(\cdot)$ is $C^{2}$ with $\Lambda^{\prime \prime}(\cdot)>0$, the latter implying strict convexity of $\Lambda(p)$. Since $\Lambda^{\prime}(\cdot)$ is continuous and strictly increasing, for any $\eta \in\left(\Lambda^{\prime}(0), \Lambda^{\prime}(1)\right)$, there is a unique $p^{*} \in(0,1)$ with $\Lambda^{\prime}\left(p^{*}\right)=\eta$. The strict convexity of $\Lambda(p)-p \eta$ and $\Lambda(p)+(1-p) \eta$ implies that the infima in (5) are attained at $p^{*}$. The negativity of $\mathscr{E}_{F}(\cdot), \mathscr{E}_{M}(\cdot)$ over $(0,1)$ can be shown using convexity. Equation (12) follows from Theorem 2.

\section{Vi. Application to Nuclear detection}

For concreteness, we consider the following scenario. We have five sensors deployed along the $x$-axis at locations $\left(x_{1}, y_{1}\right)=(-3,0),\left(x_{2}, y_{2}\right)=(-1,0),\left(x_{3}, y_{3}\right)=(0,0)$, $\left(x_{4}, y_{4}\right)=(1,0),\left(x_{5}, y_{5}\right)=(3,0)$. A potential source with intensity $a>0$ circles the point $(0,2)$ with radius $R=0.5$, moving with angular velocity $4 \pi / 5$. The coordinates of the source are thus given by

$$
x_{s}(t)=0.5 \cos \left(\frac{4 \pi t}{5}\right), y_{s}(t)=2+0.5 \sin \left(\frac{4 \pi t}{5}\right) .
$$

We assume uniform background radiation with $\beta_{i}(t) \equiv \beta$.

For $a=5$ and $\beta=13$ counts per second, $T=10$ seconds, $\chi=1 \mathrm{~m}^{2}$, Fig. 1 compares (the Monte Carlo estimates of) the error probabilities $P_{F}$ and $P_{M}$, with the corresponding Chernoff upper bounds, for various values of the threshold.

From thefigure, we see that for both $P_{F}$ and $P_{M}$, the Chernoff bounds and Monte Carlo estimates follow the same trend. In addition, the gap between the Monte Carlo estimate and the Chernoff bound, which at its maximum seems to be in the order of $75 \%$, is reduced near the boundaries of the threshold range as a result of the probability being

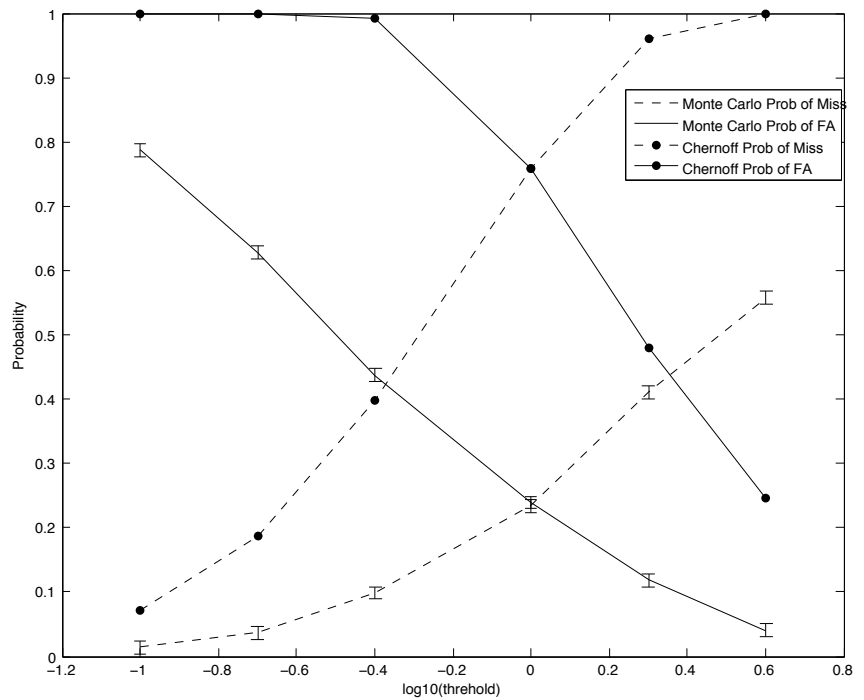

Fig. 1. The comparison between Chernoff bound and Monte Carlo simulation. Both probability of false alarm and miss are calculated for different threshold using both methods. The Chernoff bounds of probabilities are marked with black dots. The $95 \%$ confidence intervals of Monte Carlo estimations are marked with bars.

bounded in the $[0,1]$ interval. Typically in nuclear detection, the probability of false alarm is desired to be very small, in the order of $10^{-6}$ or smaller, and in that range the gap between the Monte Carlo estimates and the Chernoff bounds falls to acceptable levels.

\section{AnAlytical Threshold SELECtion}

Consider the decision problem posed in Section III with general $\beta_{i}(\cdot), \nu_{i}(\cdot), 1 \leq i \leq k$, and time horizon $T$. Theorem 1 tells us that for $\gamma>0$, the LR test $\left\{L_{T} \geq \gamma\right\}$ is optimal (in the Neyman-Pearson sense) among all tests with probability of false alarm less than or equal to $\alpha=\mathbb{P}_{0}\left(L_{T} \geq \gamma\right)$. This leads to the question: how does one select $\gamma$ to conform with the desired performance requirements?

The problem of selecting the threshold $\gamma$ is nontrivial, both analytically and numerically. From the analytic standpoint, it can be difficult to specify the distribution of $L_{T}$ - especially in a multidimensional, time-inhomogeneous problem such as ours-greatly complicating any attempts to obtain closedform expressions for the error probabilities $P_{F}, P_{M}$ defined in (2). One may opt, therefore, to numerically test various values of $\gamma$ with the goal of converging towards a choice for which $P_{F}, P_{M}$ are of the right order of magnitude. This, however, presents us with the two following problems. The first is the sizeable computational burden. Indeed, if one selects any particular $\gamma$, and uses a basic Monte Carlo method to estimate (say) $P_{F}$ to an accuracy of $10^{-3}$ (not unreasonable if the desired probability of false alarm is set at $10^{-2}$ ) with $95 \%$ confidence, then at least $\frac{1}{2 \times 10^{-6}} \ln \left(\frac{2}{0.05}\right) \approx$ 1844439.73 simulation runs are needed [29]. The second problem is that a numerical computation of error probabilities as a function of threshold, as in Fig. 1, is tied to a very specific source motion and sensor configuration. Should any of the parameters change, the numerical computation has to be repeated. 
In such instances, the Chernoff bounds provide a simple, elegant method for selecting $\eta=\log \gamma$, at the expense of some sharpness. The idea here is to use the upper bounds on $P_{F}$ and $P_{M}$ as proxies for the corresponding probabilities. One formulates the problem as follows: Suppose $\alpha \in(0,1)$ is given. Knowing the tightest bounds on $P_{F}, P_{M}$ for given $\eta$ (through Proposition 2), how should $\eta$ be chosen to minimize the tightest upper bound on $P_{M}$ while ensuring that the tightest upper bound on $P_{F}$ does not exceed $\alpha$ ? The identification of $\eta$ is thus reduced to a tractable (deterministic) constrained optimization problem.

Proposition 2 assures us that for every $\eta \in\left(\Lambda^{\prime}(0), \Lambda^{\prime}(1)\right)$, there is a unique $p^{*} \in(0,1)$ (satisfying $\Lambda^{\prime}\left(p^{*}\right)=\eta$ ) where the tightest bounds on $P_{F}, P_{M}$ for the LR test $\left\{L_{T} \geq \mathrm{e}^{\eta}\right\}$ are attained. Since there is a one-to-one correspondence between $(0,1)$ and $\left(\Lambda^{\prime}(0), \Lambda^{\prime}(1)\right)$ given by the strictly increasing continuous function $p \mapsto \Lambda^{\prime}(p)$, one can pose the search for suitable $\eta \in\left(\Lambda^{\prime}(0), \Lambda^{\prime}(1)\right)$ in terms of a search for suitable $p \in(0,1)$, with the understanding that for any $p \in(0,1)$, the corresponding $\eta$ is given by $\eta=\Lambda^{\prime}(p)$. Recalling (10a), (10b), we have the constrained minimization problem

$$
\text { minimize } \mathscr{E}_{M}(p) \text { over } p \in(0,1) \text { with } \mathscr{E}_{F}(p) \leq \log \alpha
$$

Proposition 3, stated next, says that if $\alpha$ is not too small, there is a unique $p^{\dagger} \in(0,1)$ that is the solution to (14).

\section{Proposition 3: Suppose}

$$
\log \alpha>-\Lambda^{\prime}(1)
$$

Then, there is a unique $p^{\dagger} \in(0,1)$ which solves the equation $\mathscr{E}_{F}(p)=\log \alpha$. Moreover, $p^{\dagger}$ minimizes $\mathscr{E}_{M}(p)$ over all $p \in$ $(0,1)$ which satisfy $\mathscr{E}_{F}(p) \leq \log \alpha$. This minimum value of $\mathscr{E}_{M}$ is given by $\mathscr{E}_{M}\left(p^{\dagger}\right)=\log \alpha+\Lambda^{\prime}\left(p^{\dagger}\right)$. Choosing $\eta=$ $\Lambda^{\prime}\left(p^{\dagger}\right)$, i.e. taking $\gamma=\mathrm{e}^{\Lambda^{\prime}\left(p^{\dagger}\right)}$ in the decision test $\left\{L_{T} \geq \gamma\right\}$, we have $P_{F} \leq \alpha, P_{M} \leq \alpha \mathrm{e}^{\Lambda^{\prime}\left(p^{\dagger}\right)}$.

Proof: One can show that, on the interval $(0,1), \mathscr{E}_{F}$ is strictly decreasing while $\mathscr{E}_{M}$ is strictly increasing, with

$$
\begin{array}{ll}
\inf _{p \in(0,1)} \mathscr{E}_{F}(p)=-\Lambda^{\prime}(1), & \sup _{p \in(0,1)} \mathscr{E}_{F}(p)=0, \\
\inf _{p \in(0,1)} \mathscr{E}_{M}(p)=\Lambda^{\prime}(0), & \sup _{p \in(0,1)} \mathscr{E}_{M}(p)=0 .
\end{array}
$$

Thus, in order that there exist $p \in(0,1)$ with $\mathscr{E}_{F}(p) \leq$ $\log \alpha$, it is necessary (and sufficient) that $\alpha$ satisfy $\log \alpha>$ $-\Lambda^{\prime}(1)$. With this restriction in place, there is a unique $p^{\dagger} \in$ $(0,1)$ with $\mathscr{E}_{F}\left(p^{\dagger}\right)=\log \alpha$, where we have used the fact that $\log \alpha<0$. Since $\mathscr{E}_{M}$ is strictly increasing, we see that $p^{\dagger}$ is the unique solution to the constrained minimization problem (14). As discussed, $\eta$ should be chosen to equal $\Lambda^{\prime}\left(p^{\dagger}\right)$. The bounds on $P_{F}, P_{M}$ follow from Theorem 2 .

\section{CONCLUSIONS}

For an LR test applied to networked nuclear detection where a mobile source is to be detected by a spatially distributed array of radiation sensors, the error probabilities can be conservatively estimated using Chernoff bounds. The paper provides analytic expressions for these bounds, by utilizing accepted models of remote nuclear measurement, and combining them with the form of the time-inhomogeneous statistics of radiation counts at sensor sites. These bounds are shown not to be overly conservative compared to alternative Monte Carlo-based simulation studies, and provide a pathway to the selection of threshold constants for an LR test.

\section{APPENDIX}

Proof: [Proof of Lemma 1] The result is obviously true for $p=0$. Suppose first that $p>0$. Let $K_{p} \triangleq$ $\left(1+\nu_{\max } / \beta_{\min }\right)^{p}$. Recalling Assumptions 1 and 2, we have

$$
L_{t}^{p} \leq \prod_{i=1}^{k} K_{p}^{N_{t}(i)}
$$

Thus, $\mathbb{E}_{0}\left[L_{t}^{p}\right] \leq \mathbb{E}_{0}\left[\prod_{i=1}^{k} K_{p}^{N_{t}(i)}\right]=\prod_{i=1}^{k} \mathbb{E}_{0}\left[K_{p}^{N_{t}(i)}\right]$, the latter equality following from independence. Since $N_{t}(i)$ is Poisson with intensity $\beta_{i}(t)$ under $\mathbb{P}_{0}$, one directly computes that $\mathbb{E}_{0}\left[K_{p}^{N_{t}(i)}\right]=\exp \left[\left(K_{p}-1\right) \int_{0}^{t} \beta_{i}(s) d s\right]$. Hence, $\mathbb{E}_{0}\left[L_{t}^{p}\right] \leq \exp \left[\left(K_{p}-1\right) \sum_{i=1}^{k} \int_{0}^{t} \beta_{i}(s) d s\right]<\infty$. Now suppose $p<0$. Noting that $\prod_{n=1}^{N_{t}(i)}\left(1+\frac{\nu_{i}\left(\tau_{n}(i)\right)}{\beta_{i}\left(\tau_{n}(i)\right)}\right)^{p} \leq 1$, we have

$$
L_{t}^{p} \leq \exp \left(-p \sum_{i=1}^{k} \int_{0}^{t} \nu_{i}(s) d s\right)
$$

implying that $\mathbb{E}_{0}\left[L_{t}^{p}\right]<\infty$.

Proof: [Proof of Lemma 2] Note that the result is clearly true for $p=0$. For $p \in \mathbb{R} \backslash\{0\}, t \in[0, T]$, we have

$$
L_{t}^{p}=\left(\prod_{i=1}^{k} \prod_{n=1}^{N_{t}(i)}\left[\mu_{i}\left(\tau_{n}(i)\right)\right]^{p}\right) \cdot \mathrm{e}^{p \sum_{i=1}^{k} \int_{0}^{t}\left[1-\mu_{i}(s)\right] \beta_{i}(s) d s} .
$$

Let $x(t) \triangleq \prod_{i=1}^{k} \prod_{n=1}^{N_{t}(i)}\left[\mu_{i}\left(\tau_{n}(i)\right)\right]^{p}$, and $y(t) \triangleq$ $\mathrm{e}^{p \sum_{i=1}^{k} \int_{0}^{t}\left[1-\mu_{i}(s)\right] \beta_{i}(s) d s}$, with the convention that $\prod_{n=1}^{0}(\cdots)=1$. For $t \in(0, T], 1 \leq i \leq k$, let $\Delta N_{t}(i) \triangleq N_{t}(i)-N_{t-}(i)$ be the jump in $N_{t}(i)$ at time $t$. Since $N_{t}(i)$ and $N_{t}(j)$ are independent for $i \neq j$, they have no common jumps (see Remark 3 ):

$$
\sum_{0<t \leq T} \Delta N_{t}(i) \cdot \Delta N_{t}(j)=0, \quad \mathbb{P}_{0} \text {-a.s. }
$$

Hence, for $t \in(0, T]$ and $n \geq 1$,

$$
x(t)= \begin{cases}x(t-) \mu_{i}(t)^{p} & \text { if } t=\tau_{n}(i), i \in\{1, \ldots, k\} \\ x(t-) & \text { otherwise }\end{cases}
$$

with $x(0)=1$. Thus, $x(t)$ is right-continuous, monotone (non-decreasing if $p>0$, non-increasing if $p<0$ ) and piecewise constant with $\Delta x(t) \triangleq x(t)-x(t-)$ satisfying

$$
\Delta x(t)=\sum_{i=1}^{k} \sum_{n \geq 1} x(t-)\left[\mu_{i}(t)^{p}-1\right] 1_{\left(t=\tau_{n}(i)\right)} .
$$

Note also that $y(t)$ is differentiable with $y^{\prime}(t)=$ $y(t) \cdot p \sum_{i=1}^{k}\left[1-\mu_{i}(t)\right] \beta_{i}(t)$. By the Product Formula [3, Theorem A4.T2], $x(t) y(t)=x(0) y(0)+\int_{0}^{t} x(s-) d y(s)+$ 
$\int_{0}^{t} y(s) d x(s)$. Now, $\quad \int_{0}^{t} y(s) d x(s) \quad$ is written as $\sum_{i=1}^{k} \sum_{n \geq 1} 1_{\left(\tau_{n}(i) \leq t\right)} y\left(\tau_{n}(i)\right) x\left(\tau_{n}(i)-\right)\left[\mu_{i}\left(\tau_{n}(i)\right)^{p}-1\right]=$ $\sum_{i=1}^{k} \int_{0}^{t} y(s) x(s-)\left[\mu_{i}(s)^{p}-1\right] d N_{s}(i)$, where the latter integrals are defined as follows: for a stochastic process $C_{t}$, we have $\int_{0}^{t} C_{s} d N_{s}(i) \triangleq \sum_{n \geq 1} C_{\tau_{n}(i)} 1_{\left(\tau_{n}(i) \leq t\right)}$, for $t \in[0, T], 1 \leq i \leq k$. Also, $\int_{0}^{t} x(s-) d y(s)=$ $\int_{0}^{t} x(s-) y(s) \cdot\left(p \sum_{i=1}^{k}\left[1-\mu_{i}(s)\right] \beta_{i}(s)\right) d s$.

Noting that $y(s)=y(s-)$ for all $s \in[0, T]$ (owing to the continuity of $y(\cdot)$ ), we get

$$
\begin{aligned}
L_{t}^{p}=1+\sum_{i=1}^{k} \int_{0}^{t} L_{s-}^{p}\left[\mu_{i}(s)^{p}-1\right] d N_{s}(i) \\
+p \sum_{i=1}^{k} \int_{0}^{t} L_{s-}^{p}\left[1-\mu_{i}(s)\right] \beta_{i}(s) d s
\end{aligned}
$$

Since $M_{t}(i)=N_{t}(i)-\int_{0}^{t} \beta_{i}(s) d s$, we have

$$
\begin{gathered}
\int_{0}^{t} L_{s-}^{p}\left[\mu_{i}(s)^{p}-1\right] d M_{s}(i)=\int_{0}^{t} L_{s-}^{p}\left[\mu_{i}(s)^{p}-1\right] d N_{s}(i) \\
-\int_{0}^{t} L_{s-}^{p}\left[\mu_{i}(s)^{p}-1\right] \beta_{i}(s) d s .
\end{gathered}
$$

Using the latter in equation (20) yields the stated result.

Remark 3: The claim in (18) can be verified as follows. Let $\left(\tilde{L}_{t}: t \in[0, T]\right)$ be the process

$$
\tilde{L}_{t} \triangleq \prod_{i=1}^{k}\left\{\exp \left(\int_{0}^{t}\left[\beta_{i}(s)-1\right] d s\right) \prod_{n=1}^{N_{t}(i)} \frac{1}{\beta_{i}\left(\tau_{n}(i)\right)}\right\} .
$$

Since $0<\beta_{\min } \leq \beta_{i}(t) \leq \beta_{\max }<\infty$ for all $t \in[0, T]$, $1 \leq i \leq k$, one can check that the equation $d \mathbb{Q} / d \mathbb{P}_{0}=\tilde{L}_{T}$ defines a probability measure $\mathbb{Q}$ on $(\Omega, \mathscr{F})$ such that $\mathbb{Q}$ and $\mathbb{P}_{0}$ are mutually absolutely continuous-thus $\mathbb{Q}$ is absolutely continuous with respect to $\mathbb{P}_{0}$ (denoted $\mathbb{Q} \ll \mathbb{P}_{0}$ ) and $\mathbb{P}_{0}$ is absolutely continuous with respect to $\mathbb{Q}$ (denoted $\mathbb{P}_{0} \ll \mathbb{Q}$ ) with $d \mathbb{P}_{0} / d \mathbb{Q}=1 / \tilde{L}_{T}$. Moreover, with respect to $\mathbb{Q}$, the $N_{t}(i)$ 's are independent Poisson processes with intensity 1. Since, by [30, Proposition XII.1.5], $\sum_{0<t<T} \Delta N_{t}(i)$. $\Delta N_{t}(j)=0$, $\mathbb{Q}$-a.s., we must have (18), owing to $\mathbb{P}_{0} \ll \mathbb{Q}$.

\section{REFERENCES}

[1] R. Byrd, J. Moss, W. Priedhorsky, C. Pura, G. W. Richter, K. Saeger, W. Scarlett, S. Scott, and R. W. Jr., "Nuclear detection to prevent or defeat clandestine nuclear attack," IEEE Sensors Journal, vol. 5, no. 4, pp. 593-609, 2005.

[2] R. J. Nemzek, J. S. Dreicer, D. C. Torney, and T. T. Warnock, "Distributed sensor networks for detection of mobile radioactive sources," IEEE Transactions on Nuclear Science, vol. 51, no. 4, pp. 1693-1700, 2004.

[3] P. Brémaud, Point Processes and Queues. Springer-Verlag, 1981.

[4] H. Chen and D. D. Yao, Fundamentals of Queueing Networks. Springer-Verlag, 2001

[5] R. M. Gagliardi and S. Karp, Optical Communications, second edition ed. Wiley-Interscience, 1995.

[6] H. C. Tuckwell, Stochastic Processes in the Neurosciences. SIAM, 1989.
[7] P. V. R. Boel and E. Wong, "Martingales on jump processes II: Applications," SIAM Journal on Control, vol. 13, no. 5, pp. 1022$1061,1975$.

[8] M. H. A. Davis and E. Andreadakis, "Exact and approximate filtering in signal detection: An example," IEEE Transactions on Information Theory, vol. 23, no. 6, pp. 768-772, 1977.

[9] E. A. Geraniotis and H. V. Poor, "Minimax discrimination for observed Poisson processes with uncertain rate functions," IEEE Transactions on Information Theory, vol. 31, no. 5, pp. 660-669, 1985.

[10] J. L. Hibey, D. L. Snyder, and J. H. van Schuppen, "Error-probability bounds for continuous-time decision problems," IEEE Transactions on Information Theory, vol. 24, no. 5, pp. 608-622, 1978.

[11] S. Verdú, "Asymptotic error probability of binary hypothesis testing for poisson point-process observations," IEEE Transactions on Information Theory, vol. 32, no. 1, pp. 113-115, 1986.

[12] D. Stephens Jr and A. Peurrung, "Detection of moving radioactive sources using sensor networks," IEEE Transactions on Nuclear Science, vol. 51, no. 5, pp. 2273-2278, 2004.

[13] A. Sundaresan, P. K. Varshney, and N. S. V. Rao, "Distributed detection of a nuclear radioactive source using fusion of correlated decisions," in Proceedings of the International Conference on Information Fusion. IEEE, 2007, pp. 1-7.

[14] M. Morelande, B. Ristic, and A. Gunatilaka, "Detection and parameter estimation of multiple radioactive sources," in Proceedings of the International Conference on Information Fusion. IEEE, 2007, pp. $1-7$.

[15] S. M. Brennan, A. M. Mielke, and D. C. Torney, "Radioactive source detection by sensor networks," IEEE Transactions on Nuclear Science, vol. 52, no. 3, pp. 813-819, 2005.

[16] D. L. Snyder, Random Point Processes. Wiley-Interscience, 1975.

[17] J. E. Evans, "Chernoff bounds on the error probability for the detection of non-Gaussian signals," IEEE Transactions on Information Theory, vol. 20, no. 5, pp. 569-577, 1974.

[18] C. M. Newman and B. W. Stuck, "Chernoff bounds for discriminating between two Markov processes," Stochastics, vol. 2, pp. 139-153, 1979.

[19] V. K. Prabhu, "Modified Chernoff bounds for PAM systems with noise and interference," IEEE Transactions on Information Theory, vol. 28 , no. 1, pp. 95-100, 1982.

[20] S. Verdú, "Multiple-access channels with point-process observations: Optimum demodulation," IEEE Transactions on Information Theory, vol. 32 , no. 5, pp. 642-651, 1986.

[21] J. J. O'Reilly and J. R. F. da Rocha, "Improved error probability evaluation methods for direct detection optical communication systems," IEEE Transactions on Information Theory, vol. 33, no. 6, pp. 839-848, 1987.

[22] L. B. Nelson and H. V. Poor, "Performance of multiuser detection for optical cdma-part i: Error probabilities," IEEE Transactions on Communications, vol. 43, no. 11, pp. 2803-2811, 1995.

[23] J. S. Sadowsky, "An asymptotically least-favorable Chernoff bound for a large class of dependent data processes," IEEE Transactions on Information Theory, vol. 33, no. 1, pp. 52-61, January 1987.

[24] D. Kazakos, "Asymptotic error probability expressions for multihypothesis testing using multisensor data," IEEE Transactions on Systems, Man and Cybernetics, vol. 21, no. 5, pp. 1101-1114, September/October 1991.

[25] J. A. Bucklew and J. S. Sadowsky, "A contribution to the theory of Chernoff bounds," IEEE Transactions on Information Theory, vol. 39, no. 1, pp. 249-254, 1993.

[26] C. D. Pahlajani, I. Poulakakis, and H. G. Tanner, "Networked decision making for Poisson processes: application to nuclear detection," IEEE Transactions on Automatic Control (submitted).

[27] E. L. Lehmann, Testing Statistical Hypotheses, 2nd ed. John Wiley \& Sons, 1986

[28] H. V. Poor, An Introduction to Signal Detection and Estimation, 2nd ed. Springer-Verlag, 1994.

[29] M. Vidyasagar, "Statistical learning theory and randomized algorithms for control," IEEE Control Systems Magazine, vol. 18, no. 6, pp. 6985, 1998.

[30] D. Revuz and M. Yor, Continuous Martingales and Brownian Motion, 3rd ed. Springer, 1999. 